\title{
(6) OPEN ACCESS \\ Outcomes in patients with heart failure treated in hospitals with varying admission rates: population-based cohort study
}

\author{
R Sacha Bhatia, ${ }^{1,2,3,4}$ Peter C Austin, ${ }^{1,3}$ Therese A Stukel, ${ }^{1,3}$ \\ Michael J Schull, ${ }^{1,4,5}$ Alice Chong, ${ }^{1}$ Jack V Tu, ${ }^{1,3,4,6}$ Douglas S Lee ${ }^{1,3,4,7}$
}

- Additional material is published online only. To view please visit the journal online (http://dx.doi.org/10.1136/bmjqs2014-002816)

For numbered affiliations see end of article.

\section{Correspondence to}

Dr Douglas S Lee, Senior Scientist, Institute for Clinical Evaluative Sciences, Division of Cardiology, University Health Network, Associate Professor of Medicine, University of Toronto, ICES, Rm G-106, 2075 Bayview Avenue, Toronto, Ontario, Canada M4N 3M5; dlee@ices.on.ca

Received 7 January 2014 Revised 15 June 2014 Accepted 17 June 2014 Published Online First 31 July 2014

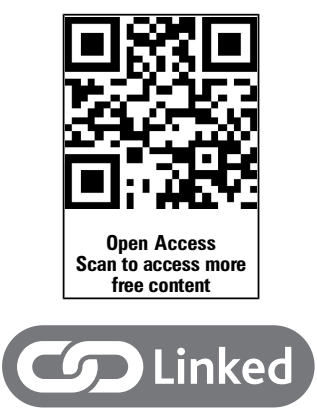

- http://dx.doi.org/10.1136/ bmjqs-2014-003586

\section{CrossMark}

To cite: Bhatia RS,

Austin PC, Stukel TA, et al.

BMJ Qual Saf 2014;23:

981-988

\section{ABSTRACT}

Study objectives Hospital admission rates for patients with heart failure (HF) presenting for emergency department (ED) care vary, and the implications of direct discharge home from the ED are unknown. We examined whether patients treated in hospitals with low admission rates exhibit higher rates of repeat ED visits, hospital readmissions and death.

Methods We divided EDs into low-, mediumand high-admission-rate tertiles by their standardised HF admission rate in Ontario, Canada. Among adults ( $\geq 18$ years) with HF discharged from an ED between April 2004 and March 2010, we evaluated the primary outcomes of repeat ED visits or hospitalisations for $\mathrm{HF}$, and secondary outcomes, which included death, within 30 days stratified by HF admission-rate tertile.

Results 89878 patients with HF presented to low- ( $n=29$ 929), medium- ( $n=30900)$ or high( $n=29049$ ) admission-rate institutions, with hospitalisation rates of $<67 \%, 67-75 \%$ and $>75 \%$, respectively. Among 28175 EDdischarged patients, the multivariable-adjusted $\mathrm{HR}$ for repeat ED visit or hospitalisation for HF at low-admission-rate institutions was $1.18(95 \% \mathrm{Cl}$ 1.07 to $1.29, p<0.001$ ) compared with highadmission institutions. Similarly, the HR for repeat ED visits for HF was 1.28 (95\% Cl 1.14 to $1.44, \mathrm{p}<0.001)$ at low-admission hospitals. Compared with discharged patients in the highadmission-rate tertile, adjusted HR for 30-day mortality was 1.19 (95\% Cl 0.95 to 1.47$)$ at lowadmission-rate hospitals. The HRs for all of the above outcomes were not significantly increased at medium-admission-rate hospitals

Discussion Patients seeking care at institutions with lower rates of HF admission showed higher rates of repeat ED visits or hospitalisations after previous ED discharge.

\section{INTRODUCTION}

Heart failure (HF) is associated with substantial morbidity and high rates of hospital admission and readmission. Consequently, a large proportion of the costs of HF care are incurred by patients who receive care in the hospital setting. ${ }^{12}$ The health system impact of HF admissions has been a focus of system-wide quality improvement efforts world-wide. High rates of $\mathrm{HF}$ readmission prompted its use as a performance metric for hospitals, and the Center for Medicare and Medicaid Services in the United States began publicly reporting on 30-day readmission rates in 2009. ${ }^{3} 4$ Subsequent evaluation demonstrated that readmission rates are complex metrics that may be affected by system-level factors. ${ }^{5}$

There is substantial economic burden of $\mathrm{HF}$ admissions and a focus on readmissions as a quality of care measure. ${ }^{6}$ However, there is little appreciation of the pitfalls of using readmissions as a quality metric and the system factors that contribute to variations in hospitalisations. For example, lower regional admission rates have been associated with reduced $\mathrm{HF}$ readmission rates, ${ }^{5}$ and it is unknown if this ecological relationship extends to hospitals. Understanding variations in hospitalisations and resultant outcomes requires improved knowledge of admission decisions that are made in the emergency department (ED), since it is most often the point of initial contact with the healthcare system and the portal of entry into the hospital. ${ }^{7}$ Since risk stratification is not routinely used in emergency care of acute HF, it is possible that greater numbers of patients 
discharged from the ED may result in more return hospital visits and admissions.

In this study we explored whether the hospitalisation rate of patients with HF presenting to the ED varies across EDs in a single-payer health system. Furthermore, we determined whether patients with HF discharged from the ED, who were treated at hospitals with lower rates of admission, experienced higher rates of adverse outcomes. We hypothesised that patients cared for and discharged from EDs with lower hospital admission rates would exhibit higher rates of early repeat emergency visits and hospitalisations for recurrent HF.

\section{METHODS}

\section{Selection of participants and hospitals}

We conducted a retrospective cohort study of patients (aged $\geq 18$ years) who visited an ED in Ontario, Canada, for HF between 1 April 2004 and 31 March 2010. Included patients were Ontario residents with a valid health card number. To determine crude admission rates, we calculated the proportion of patients with $\mathrm{HF}$ admitted relative to all patients who visited the $\mathrm{ED}$ for $\mathrm{HF}$ at each institution. To evaluate the impact of hospital admission rate on outcome, we examined patients who were discharged home from the ED since this group is at risk of early repeat hospital visits and death. ${ }^{8}$ We excluded patients with HF that were admitted to hospital by any route other than the ED (ie, inter-hospital transfer). Institutions with fewer than $20 \mathrm{HF}$ visits to the ED annually and urgent care centres (ie, ambulatory care centres that treat patients seeking emergency care but do not have on-site acute care hospital beds) were excluded. Urgent care centres were included in a sensitivity analysis. Research ethics board approval was obtained from Sunnybrook Health Sciences Centre.

\section{Outcomes}

The primary outcomes were HF-specific, since readmissions for HF after an initial ED discharge for the same condition may indicate poor quality of care. The primary outcome was the occurrence of repeat ED visits or hospitalisations for $\mathrm{HF}$ within 30 days after the initial ED discharge. The co-primary outcome was repeat ED visits for HF. Secondary outcomes included 30-day mortality and the composite of (i) ED visits or hospitalisations for cardiovascular disease and (ii) allcause ED visits and hospitalisations. We also examined whether physician care, use of diagnostic tests, and revascularisation procedures were differentially performed after discharge from low-, medium- or high-admission-rate EDs.

\section{Data sources}

Multiple administrative databases were linked deterministically by using each patient's unique, encrypted health card number. We used the National
Ambulatory Care Reporting System (NACRS) database, which contains information on all ED visits, to identify patients with HF seeking emergency care. Patients with HF were identified using the International Classification of Diseases 10th revision ICD-10-CA code I50; validation studies have found high predictive value of HF diagnostic codes compared with the Framingham criteria. ${ }^{9}$ Hospital admissions were identified using the Canadian Institute for Health Information Discharge Abstract Database (CIHI-DAD). In the case of multiple emergency records, only the first visit was counted as the index encounter for the purposes of determining repeat emergency visits and hospitalisations.

The Ontario Health Insurance Plan (OHIP) claims database and the CIHI-DAD were used to identify diagnostic or interventional cardiac procedures performed within 30 days after emergency discharge, while the former was used to identify physician visits. Physician visits and procedures are billed on the date of service, with random audits conducted by the Ministry of Health and Long-Term Care, the single payer of health services. Physician specialty was determined from the Ontario Physician Human Resource Data Centre database. Physician specialty was defined by the 'functional specialty' determined, via telephone survey, by the type of care constituting the majority of the physician's practice. Therefore, internal medicine specialists who provided primarily cardiology care were also considered cardiac specialists. ${ }^{10}$ Performance of the following procedures was examined as markers of patient care: (i) testing of left ventricular ejection fraction by echocardiography or radionuclide angiography; (ii) stress testing; (iii) cardiac catheterisation; and (iv) coronary revascularisation, including percutaneous coronary intervention or coronary artery bypass graft surgery (procedure codes shown in online supplementary appendix 1). Deaths were determined from the Registered Persons Database.

\section{Statistical analysis}

The distributions of continuous variables were compared across hospital admission categories using the Kruskal-Wallis test, and categorical variables were compared using the Mantel-Haenszel $\chi^{2}$ test for trend. The age- and sex-standardised hospital HF admission rate was the primary exposure variable and was calculated as follows:

$$
\begin{gathered}
\text { Age } / \text { sex }- \text { standardised hospital HF admission rate } \\
=(\text { Observed } \# \text { of patients admitted } / \text { Expected } \\
\# \text { of patients admitted }) \times \text { Crude admission rate }
\end{gathered}
$$

where the expected number of admitted patients was the sum of the product of the number of patients in each age/sex stratum in the hospital and the crude 
admission rate in each age/sex stratum in the overall cohort. We divided institutions into low-, mediumand high-admission-rate tertiles based on the standardised hospital admission rate, with a similar number of patients per tertile as performed previously. ${ }^{11} 12$

Age/sex-adjusted event rates were reported as rates per person-time and compared using Cox proportional hazards regression analysis. The effect of admission-rate tertile on time-to-event outcomes was evaluated using multiple Cox proportional hazards analyses, with adjustment for previously published HF-specific risk-adjustment models for prediction of death or morbidity (see online supplementary appendices 2 and 3). ${ }^{13}{ }^{14}$ We also adjusted for hospital type (small community, large community or academic hospital), rurality and residential neighbourhood income quintile. Finally, we adjusted for visit-specific factors, such as time of presentation (eg, daytime 08:00-17:00, evening 17:01-24:00, or morning 00:01-07:59), ED length of stay, chief complaint (eg, suspected HF, dyspnoea), number of previous ED visits for HF in past 3 years, and the Canadian Triage Acuity Score, which signified the acuity of the emergency patient as judged by the nurse at triage. For each outcome, including repeat ED visits and hospitalisations for HF, patients were censored at 30 days or upon death, whichever occurred earlier if the event of interest had not yet occurred. Comorbidities were determined by examining all diagnosis codes in the CIHI-DAD in the 3 years before the index ED visit. A summary-predicted tertile-specific 30-day mortality rate was calculated using multiple logistic regression, with adjustment for the clinical covariates and ED-specific factors described above. Analyses were performed at the patient level, clustered hierarchically within EDs. The Cox regression models used robust, sandwich-type variance estimators to account for the clustering of patients within EDs. ${ }^{15}$

We determined if patients discharged from mediumand low-admission-rate hospitals were less likely to receive care by any physician, family physician or cardiac specialist or received shared care (family physician + cardiac specialist) than high-admission-rate hospitals within 30 days using previously published methods. ${ }^{10}$ We also examined diagnostic tests and revascularisation procedures using multiple Cox regression analysis to compare differences after ED discharge. All of the above analyses were adjusted for the risk-adjustment variables predictive of morbidity and mortality shown in online supplementary appendix $3,{ }^{13} 14$ and the ED-specific factors listed above. Statistical significance was indicated by a two-tailed $\mathrm{p}$ value $<0.05$. All analyses were performed with SAS V.9.2.

\section{RESULTS}

\section{Characteristics of study subjects}

After identification of unique ED visits (figure 1), 89878 patients remained, of whom 28175 (31.3\%) were discharged and $61703(68.7 \%)$ were admitted. The discharged patients comprising the primary cohort presented to one of $162 \mathrm{EDs}$, and were divided into tertiles based on institutional admission rates. The median (25th, 75 th centiles) HF hospital admission rates for low-, medium- and high-rate tertiles were $57.9 \%(51.2,62.2),. 70.2 \%(67.8,73.7)$ and $78.5 \%(76.3,81.4)$, respectively. There was higher predicted probability of 30-day mortality for all patients with HF who presented to an ED at highand medium- (reflecting higher-risk case mix) than low-admission-rate institutions (table $1, \mathrm{p}<0.001$ ).

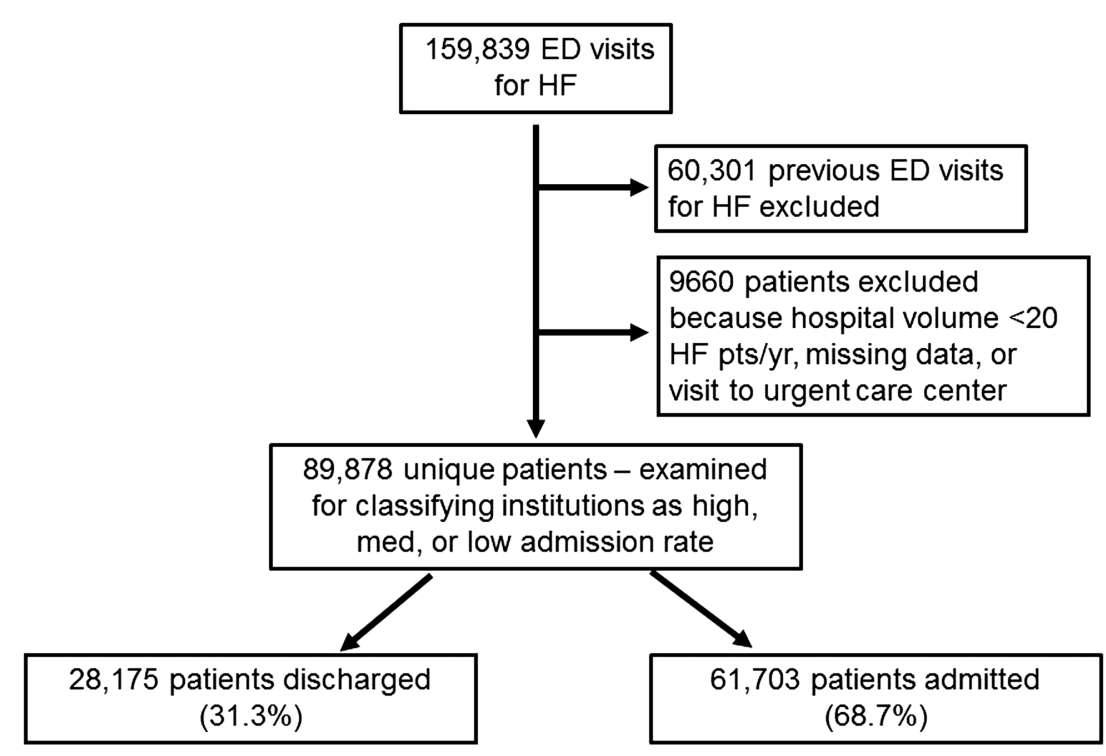

Figure 1 Study cohort flow diagram. 
Original research

Table 1 Patient characteristics by admission tertile

\begin{tabular}{|c|c|c|c|c|}
\hline Characteristic & High admission rate & Medium admission rate & Low admission rate & p Value \\
\hline $\mathrm{N}$ & 29049 & 30900 & 29929 & \\
\hline Age (years) & $79(70,85)$ & $78(69,85)$ & $78(69,85)$ & $<0.001$ \\
\hline Male & 14209 (48.9) & $15456(50.0)$ & $15101(50.5)$ & $<0.001$ \\
\hline Myocardial infarction* & $4983(17.2)$ & $5245(17.0)$ & $4740(15.8)$ & $<0.001$ \\
\hline Prior HF hospitalisation* & $8325(28.7)$ & $9093(29.4)$ & $8456(28.3)$ & 0.27 \\
\hline Prior coronary revascularisation* & $2072(7.1)$ & $2179(7.1)$ & $2127(7.1)$ & 0.91 \\
\hline Prior implantable defibrillator* & $435(1.5)$ & $433(1.4)$ & $357(1.2)$ & 0.001 \\
\hline Prior pacemaker & $1247(4.3)$ & $1218(3.9)$ & $1021(3.4)$ & $<0.001$ \\
\hline Valvular heart disease & $1894(6.5)$ & $2032(6.6)$ & $1786(6.0)$ & 0.006 \\
\hline Hypertension & $8864(30.5)$ & $9265(30.0)$ & $7482(25.0)$ & $<0.001$ \\
\hline Atherosclerotic disease & $10677(36.8)$ & $11257(36.4)$ & $10586(35.4)$ & $<0.001$ \\
\hline Diabetes & $7434(25.6)$ & $7812(25.3)$ & $7226(24.1)$ & $<0.001$ \\
\hline Atrial fibrillation & 5708 (19.6) & $6211(20.1)$ & $5508(18.4)$ & $<0.001$ \\
\hline Ventricular arrhythmias & $525(1.8)$ & $538(1.7)$ & $461(1.5)$ & 0.012 \\
\hline Renal disease & $3514(12.1)$ & $3638(11.8)$ & $3070(10.3)$ & $<0.001$ \\
\hline CTAS & $2(2,3)$ & $2(2,3)$ & $3(2,3)$ & $<0.001$ \\
\hline \multicolumn{5}{|l|}{ Time of ED visit } \\
\hline Day (08:00-17:00) & $15969(55.0)$ & $17512(56.7)$ & $17819(59.5)$ & $<0.001$ \\
\hline Evening (17:01-24:00) & $7214(24.8)$ & $7498(24.3)$ & 6766 (22.6) & \\
\hline Morning (00:01-07:59) & $5866(20.2)$ & $5890(19.1)$ & $5344(17.9)$ & \\
\hline Emergency length of stay (h) & $5.9(3.9,8.6)$ & $5.5(3.4,8.6)$ & $4.3(2.6,7.1)$ & $<0.001$ \\
\hline \multicolumn{5}{|l|}{ Chief complaint } \\
\hline Suspected HF & $629(2.2)$ & $1615(5.2)$ & $1328(4.4)$ & 0.34 \\
\hline Dyspnoea & $19031(65.5)$ & $19455(63.0)$ & $18817(62.9)$ & \\
\hline Ankle oedema & $940(3.2)$ & $1106(3.6)$ & $1198(4.0)$ & \\
\hline Chest pain & $3425(11.8)$ & $3398(11.0)$ & $2882(9.6)$ & \\
\hline Predicted 30-day mortality & $7.9(4.7,13.0)$ & $7.4(4.4,12.2)$ & $6.7(4.0,11.1)$ & $<0.001$ \\
\hline
\end{tabular}

Values are $\mathrm{n}(\%)$ or median (25th, 75th centile).

*Occurring within 3 years before ED visit date.

CTAS, Canadian Triage Acuity Score; ED, emergency department; HF, heart failure.

\section{Hospitals}

Low-admission-rate EDs were less likely to be teaching institutions and had fewer acute care and intensive care unit beds (table 2). High-admission-rate institutions were more likely to be larger urban, community or teaching hospitals with greater numbers of acute care and intensive care unit beds.

\section{Outcomes}

Age- and sex-adjusted 30-day event rates for patients discharged from the ED in each of the three admission-rate tertiles are shown in figure 2 . The composite 30-day age/sex-adjusted event rates were highest at low-admission-rate hospitals and were driven by repeat ED visits for $\mathrm{HF}$.

Of the 28175 patients who were initially discharged without hospitalisation, 3001 (10.7\%) revisited the ED for $\mathrm{HF}$ within 30 days, with $87.6 \%$ returning to the same institution. When initially discharged patients returned to the same ED $(n=2628)$, the admission rates for the repeat visit remained lowest at low- and highest at high-admission-rate

Table 2 Hospital characteristics by admission-rate tertile

\begin{tabular}{|c|c|c|c|c|}
\hline Characteristic & High admission rate & Medium admission rate & Low admission rate & $\mathrm{p}$ Value \\
\hline$\overline{\mathrm{N}}$ & 30 & 40 & 92 & \\
\hline \multicolumn{5}{|l|}{ Hospital type } \\
\hline Community & $23(76.7)$ & $33(82.5)$ & $42(45.7)$ & 0.04 \\
\hline Teaching & $\leq 5(13.3)$ & $6(15.0)$ & $\leq 5(5.4)$ & \\
\hline Urban hospital location & $27(90.0)$ & $36(90.0)$ & $31(33.7)$ & $<0.001$ \\
\hline Total number of beds, median & $200(144,288)$ & $129(56,271)$ & $25(15,49)$ & $<0.001$ \\
\hline Number of ICU beds, median & $18(10,27)$ & $13(6,21)$ & $0(0,4)$ & $<0.001$ \\
\hline
\end{tabular}

Values are $\mathrm{n}(\%)$ or median (25th, 75 th centile).

ICU, intensive care unit. 


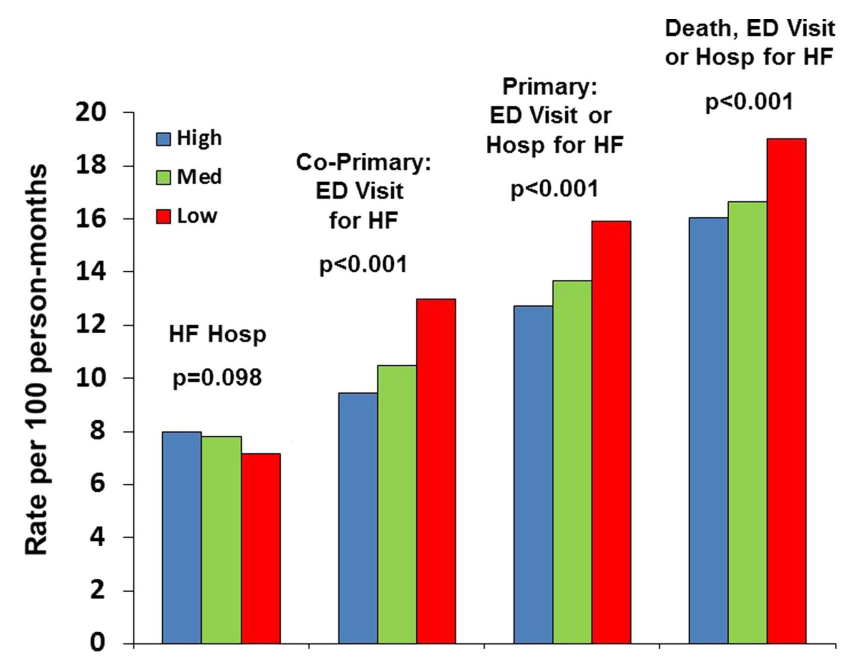

Figure 2 Age/sex-adjusted outcomes at 30 days for discharged patients by admission-rate (blue, high; green, medium; red, low) tertile. ED, emergency department; HF, heart failure.

institutions, with second-visit admission rates of $40.8 \%, 57.5 \%$ and $66.4 \%(\mathrm{p}<0.001)$. Of the 373 initially discharged patients who sought care in a different ED, there were similar gradients but higher rates of admission overall: $54.5 \%$ vs $71.6 \%$ vs $82.2 \%$ $(\mathrm{p}<0.001)$.

After adjustment for multiple covariates including patient and hospital characteristics, patients discharged from low-admission-rate EDs had a higher risk of the primary outcome of repeat emergency visits or hospitalisations for $\mathrm{HF}$ at 30 days (table 3). Compared with visiting a high-admission-rate hospital, the relative HR of the primary outcome was 1.06 (95\% CI 0.96 to $1.16, p=0.25$ ) for those discharged from medium-admission-rate institutions and $1.18(95 \%$ CI 1.07 to $1.29, \mathrm{p}<0.001)$ for those discharged from low-admission-rate institutions.

In secondary analyses, patients discharged from low-admission-rate institutions were also more likely to experience repeat ED visits and hospitalisations for cardiovascular disease and for all causes (table 3). The adjusted 30-day mortality risks were similar for admitted and discharged patients at low-admission-rate hospitals. Among admitted patients, adjusted HRs for 30-day mortality were 1.11 (95\% CI 0.99 to 1.24 , $\mathrm{p}=0.07)$ for low- and 1.06 (95\% CI 0.95 to 1.18 , $\mathrm{p}=0.29$ ) for medium- compared with high-admission-rate hospitals (referent). Among discharged patients, adjusted HRs for 30-day mortality were 1.19 (95\% CI 0.95 to $1.47, \mathrm{p}=0.12)$ for lowand $0.97(95 \%$ CI 0.78 to $1.21, \mathrm{p}=0.81)$ for medium- compared with high-admission-rate hospitals.

The likelihood of any physician visit within 30 days after discharge was not significantly different at medium- and low- compared with high-admission-rate hospitals (table 4). However, patients treated at medium- and low-admission-rate hospitals were less likely to be assessed by a cardiac specialist and less likely to experience shared care after discharge than those with high admission rates. Patients discharged from low-admission-rate institutions were less likely to undergo assessment of left ventricular function and

Table 3 Effect of HF admission rate on repeat emergency visits and hospitalisations at 30 days

\begin{tabular}{|c|c|c|}
\hline $\begin{array}{l}\text { Hospital admission-rate } \\
\text { tertile }\end{array}$ & $\begin{array}{l}\text { Adjusted HR } \\
(95 \% \mathrm{CI})\end{array}$ & $\mathrm{p}$ Value \\
\hline \multicolumn{3}{|c|}{ ED visit or hospitalisation for HF (primary outcome) } \\
\hline High & Referent & Referent \\
\hline Medium & $1.06(0.96$ to 1.16$)$ & 0.25 \\
\hline Low & $1.18(1.07$ to 1.29$)$ & $<0.001$ \\
\hline \multicolumn{3}{|c|}{ ED visit for HF (co-primary outcome) } \\
\hline High & Referent & Referent \\
\hline Medium & $1.09(0.98$ to 1.23$)$ & 0.13 \\
\hline Low & $1.28(1.14$ to 1.44$)$ & $<0.001$ \\
\hline \multicolumn{3}{|c|}{ ED visit or hospitalisation for cardiovascular disease } \\
\hline High & Referent & Referent \\
\hline Medium & $1.11(1.02$ to 1.20$)$ & 0.01 \\
\hline Low & $1.17(1.08$ to 1.26$)$ & $<0.001$ \\
\hline \multicolumn{3}{|c|}{ ED visit or hospitalisation for all causes } \\
\hline High & Referent & Referent \\
\hline Medium & $1.07(1.00$ to 1.14$)$ & 0.06 \\
\hline Low & $1.16(1.09$ to 1.23$)$ & $<0.001$ \\
\hline \multicolumn{3}{|c|}{ ED visit for cardiovascular disease } \\
\hline High & Referent & Referent \\
\hline Medium & $1.11(1.00$ to 1.23$)$ & 0.04 \\
\hline Low & $1.24(1.12$ to 1.37$)$ & $<0.001$ \\
\hline \multicolumn{3}{|l|}{ ED visit for all causes } \\
\hline High & Referent & Referent \\
\hline Medium & 1.07 (1.00 to 1.15$)$ & 0.06 \\
\hline Low & $1.17(1.09$ to 1.25$)$ & $<0.001$ \\
\hline
\end{tabular}


stress testing than those discharged from high-admission-rate institutions (table 4).

We conducted a sensitivity analysis, and found that the study findings were robust when urgent care centres were included in the analysis (see online supplementary appendix 4 and appendix figure A).

\section{DISCUSSION}

The decision to admit or discharge a patient with HF from the ED is a significant clinical challenge for physicians. In the absence of acute risk stratification for HF, emergent decisions pertaining to hospitalisation are based primarily on the physician's perception of the clinical status of the patient. ${ }^{16} 17$ In this study, we found that institutions varied in their threshold to admit patients with HF presenting to the ED irrespective of whether the visit was an initial or repeat

Table 4 Physician visits, diagnostic and therapeutic procedures within 30 days of ED discharge

\begin{tabular}{|c|c|c|}
\hline $\begin{array}{l}\text { Hospital admission } \\
\text { rate tertile }\end{array}$ & Adjusted HR (95\% Cl) & $\mathrm{p}$ Value \\
\hline \multicolumn{3}{|l|}{ Any physician visit } \\
\hline High & Referent & Referent \\
\hline Medium & $0.95(0.87$ to 1.03$)$ & 0.21 \\
\hline Low & $0.93(0.86$ to 1.01$)$ & 0.07 \\
\hline \multicolumn{3}{|c|}{ Primary care physician visit } \\
\hline High & Referent & Referent \\
\hline Medium & $0.97(0.90$ to 1.05$)$ & 0.44 \\
\hline Low & $0.98(0.90$ to 1.06$)$ & 0.62 \\
\hline \multicolumn{3}{|l|}{ Cardiac specialist visit } \\
\hline High & Referent & Referent \\
\hline Medium & $0.83(0.71$ to 0.96$)$ & 0.013 \\
\hline Low & 0.81 (0.68 to 0.95$)$ & 0.012 \\
\hline \multicolumn{3}{|l|}{ Shared care visit } \\
\hline High & Referent & Referent \\
\hline Medium & $0.83(0.71$ to 0.97$)$ & 0.018 \\
\hline Low & $0.82(0.70$ to 0.97$)$ & 0.019 \\
\hline \multicolumn{3}{|c|}{ Left ventricular function assessment } \\
\hline High & Referent & Referent \\
\hline Medium & $0.93(0.83$ to 1.05$)$ & 0.25 \\
\hline Low & 0.85 (0.75 to 0.97$)$ & 0.013 \\
\hline \multicolumn{3}{|l|}{ Stress testing } \\
\hline High & Referent & Referent \\
\hline Medium & 0.91 (0.68 to 1.22$)$ & 0.53 \\
\hline Low & $0.67(0.52$ to 0.86$)$ & 0.002 \\
\hline \multicolumn{3}{|l|}{ Cardiac catheterisation } \\
\hline High & Referent & Referent \\
\hline Medium & 1.16 (0.88 to 1.53$)$ & 0.30 \\
\hline Low & $0.87(0.63$ to 1.20$)$ & 0.39 \\
\hline \multicolumn{3}{|c|}{ Revascularisation procedure } \\
\hline High & Referent & Referent \\
\hline Medium & 0.90 (0.53 to 1.55$)$ & 0.71 \\
\hline Low & 0.71 (0.39 to 1.29$)$ & 0.27 \\
\hline
\end{tabular}

presentation. Patients who were discharged from low-admission-rate institutions experienced the highest rates of early repeat ED visits or hospitalisations.

While there have been an increasing number of studies pertaining to HF readmissions, few have examined hospital and patient level factors in conjunction with emergency visits. Hospitalised cohorts were reported in the ADHERE registry and the OPTIMIZE-HF study, and high-risk patient characteristics that predict adverse outcomes were identified. ${ }^{18} 19$ Other studies focused on hospitalised patients who were readmitted after in-hospital treatment ${ }^{620}$ or provided an incomplete picture of systemwide HF patient flow. ${ }^{21} 22$ While the overall themes were consistent, our study approached this issue in a novel way by examining hospital-specific rates of HF admission among patients presenting to the ED, emphasising the need for institutions to re-examine their rationale for admission-discharge decisions for acute HF.

Hospital readmissions exhibit complexity that is not elucidated. Previous studies have demonstrated that the risk of death increases progressively with each HF hospitalisation. ${ }^{23}{ }^{24}$ As suggested by our study, the threshold to admit may be an inherent institutional characteristic which may partly explain variations in hospitalisation rates. Indeed, initially discharged patients who presented to the same ED within 30 days for HF were more likely to be discharged again at low-admission-rate institutions. During the time period of our study, admission decisions for HF were not based on formal prognostic risk stratification, ${ }^{25}$ and early post-discharge collaborative care, which is associated with improved patient outcomes and processes of care, was not systematically employed. ${ }^{10}$

There were differences in hospital characteristics between admission-rate tertiles. Low-admission-rate hospitals had fewer acute care and intensive care unit beds than medium- and high-admission-rate hospitals. However, bed availability was unlikely to fully explain our findings, since the decision to admit patients to hospital depends on perceived clinical need rather than maintaining high occupancy rates. Low-admission-rate hospitals tended to be in nonurban areas, where specialty care may not be as accessible as in urban regions, which may affect the quality of HF management. Furthermore, while collaborative care by primary care physicians and cardiac specialists early after discharge improves HF outcomes, ${ }^{10}$ this form of management also occurred less frequently after discharge from low-admission-rate hospitals. The lack of rapid post-ED care may have partly explained the higher rates of repeat ED visits observed in this study. It is also possible that more immediate access to rapid response transitional care clinics and allied healthcare that stresses the importance of medication adherence, sodium and fluid restriction, smoking cessation and self-care may reduce ED visits. ${ }^{26} 27$ 
This study has significant clinical and public policy implications. It suggests that the outcomes experienced by patients who are discharged from the ED should not be ignored in quality-of-care evaluations. Discharge of non-low-risk patients with HF from the ED reflects poor care quality despite paradoxically improving hospitals' admission or readmission statistics. Our study supports the need for broader use of clinical risk stratification tools to prognosticate patients who present to the ED with acute $\mathrm{HF}^{7}{ }^{28}{ }^{29}$ Use of validated risk stratification tools, such as the Emergency Heart failure Mortality Risk Grade (EHMRG, https://ehmrg.ices.on. ca) ${ }^{30}$ may reduce variations by identifying (a) low-risk patients who could potentially be discharged home, and (b) higher-risk patients who are likely to experience early adverse outcomes if discharged from the ED. Adverse events experienced by emergency-discharged patients may be contributed by defects in decisionmaking in the ED. However, our findings also support the growing notion that post-hospital outcomes may also be affected by health system factors such as hospital resource availability and post-discharge transitional care.

\section{LIMITATIONS}

Our study should be interpreted in the context of its limitations. We used large, administrative databases that did not include clinical information such as blood pressure, echocardiographic data or therapy provided. However, the system-wide, population-based data available in large administrative databases are ideal for broad examinations of health systems and outcomes. In addition, we were unable to assess some hospitalspecific factors, such as presence of a cardiology service, informal referral networks between hospitals, and availability of ambulatory HF services within the community.

\section{CONCLUSION}

In conclusion, HF admission rates exhibited variability between institutions, and patients who presented to EDs with low admission rates experienced higher rates of subsequent adverse outcomes in a single-payer health system. In the absence of systematic approaches to ED-based risk stratification and well-established post-discharge ambulatory transitional care, lower rates of $\mathrm{HF}$ admission were associated with more repeat hospital visits. The use of systematic methods, such as validated risk scores, may reduce interinstitutional variations in $\mathrm{HF}$ admission rates and improve the quality of post-ED care.

\footnotetext{
Author affiliations

${ }^{1}$ Institute for Clinical Evaluative Sciences, Toronto, Ontario, Canada

${ }^{2}$ Women's College Hospital Institute for Health Systems

Solutions and Virtual Care, University of Toronto, Toronto, Ontario, Canada

${ }^{3}$ Institute of Health Policy, Management and Evaluation, University of Toronto, Toronto, Ontario, Canada
}

${ }^{4}$ Department of Medicine, Faculty of Medicine, University of Toronto, Toronto, Ontario, Canada

${ }^{5}$ Department of Emergency Medicine, Sunnybrook Health Sciences Centre, University of Toronto, Toronto, Ontario, Canada

${ }^{6}$ Division of Cardiology, Sunnybrook Health Sciences Centre, University of Toronto, Toronto, Ontario, Canada

${ }^{7}$ Peter Munk Cardiac Centre and the Joint Department of Medical Imaging of the University Health Network-Toronto

General Hospital, University of Toronto, Toronto, Ontario, Canada

Contributors All authors participated in the conception, design and interpretation of data. RSB and DSL led the drafting of the article, but all authors participated in critical revisions and granted final approval of the submitted version.

Funding Supported by operating grant MOP 114937 from the Canadian Institutes of Health Research (CIHR), Career Investigator Awards from the Heart and Stroke Foundation of Ontario (PCA, JVT), a Canada Research Chair in Health Services Research (JVT), and a CIHR Clinician-Scientist Award (DSL).

Competing interests None.

Ethics approval Sunnybrook Research Ethics Board.

Provenance and peer review Not commissioned; internally peer reviewed.

Open Access This is an Open Access article distributed in accordance with the Creative Commons Attribution Non Commercial (CC BY-NC 3.0) license, which permits others to distribute, remix, adapt, build upon this work noncommercially, and license their derivative works on different terms, provided the original work is properly cited and the use is non-commercial. See: http://creativecommons.org/licenses/by$\mathrm{nc} / 3.0 /$

\section{REFERENCES}

1 Krumholz HM, Parent EM, Tu N, et al. Readmission after hospitalization for congestive heart failure among Medicare beneficiaries. Arch Intern Med 1997;157:99-104.

2 Roger VL, Go AS, Lloyd-Jones DM, et al. Heart disease and stroke statistics-2012 update: a report from the American Heart Association. Circulation 2012;125:e2-e220.

3 Senate and House of Representatives of the United States of America in Congress. An Act Entitled The Patient Protection and Affordable Care Act. Pub L 111-148. 2010.

4 Desai AS, Stevenson LW. Rehospitalization for heart failure: predict or prevent? Circulation 2012;126:501-6.

5 Epstein AM, Jha AK, Orav EJ. The relationship between hospital admission rates and rehospitalizations. N Engl J Med 2011;365:2287-95.

6 Jencks SF, Williams MV, Coleman EA. Rehospitalizations among patients in the Medicare fee-for-service program. N Engl J Med 2009;360:1418-28.

7 Weintraub NL, Collins SP, Pang PS, et al. Acute heart failure syndromes: emergency department presentation, treatment, and disposition: current approaches and future aims: a scientific statement from the American Heart Association. Circulation 2010;122:1975-96.

8 Lee DS, Schull MJ, Alter DA, et al. Early deaths in patients with heart failure discharged from the emergency department: a population-based analysis. Circ Heart Fail 2010;3:228-35.

9 Lee DS, Donovan L, Austin PC, et al. Comparison of coding of heart failure and comorbidities in administrative and clinical data for use in outcomes research. Med Care 2005;43:182-8.

10 Lee DS, Stukel TA, Austin PC, et al. Improved outcomes with early collaborative care of ambulatory heart failure patients discharged from the emergency department. Circulation 2010;122:1806-14. 
11 Birkmeyer JD, Siewers AE, Finlayson EV, et al. Hospital volume and surgical mortality in the United States. N Engl J Med 2002;346:1128-37.

12 Harrison EM, O’Neill S, Meurs TS, et al. Hospital volume and patient outcomes after cholecystectomy in Scotland: retrospective, national population based study. BMJ 2012;344:e3330.

13 Keenan PS, Normand SL, Lin Z, et al. An administrative claims measure suitable for profiling hospital performance on the basis of 30-day all-cause readmission rates among patients with heart failure. Circ Cardiovasc Qual Outcomes 2008;1:29-37.

14 Krumholz HM, Wang Y, Mattera JA, et al. An administrative claims model suitable for profiling hospital performance based on 30-day mortality rates among patients with heart failure. Circulation 2006;113:1693-701.

15 Lin DY, Wei LJ. The robust inference for the proportional hazards model. J Am Stat Assoc 1989;84:1074-8.

16 Adams KF Jr, Fonarow GC, Emerman CL, et al. Characteristics and outcomes of patients hospitalized for heart failure in the United States: rationale, design, and preliminary observations from the first 100,000 cases in the Acute Decompensated Heart Failure National Registry (ADHERE). Am Heart J 2005;149:209-16.

17 Brophy JM, Deslauriers G, Rouleau JL. Long-term prognosis of patients presenting to the emergency room with decompensated congestive heart failure. Can J Cardiol 1994;10:543-7.

18 Fonarow GC, Adams KF Jr, Abraham WT, et al. Risk stratification for in-hospital mortality in acutely decompensated heart failure: classification and regression tree analysis. JAMA 2005;293:572-80.

19 Gheorghiade M, Abraham WT, Albert NM, et al. Systolic blood pressure at admission, clinical characteristics, and outcomes in patients hospitalized with acute heart failure. JAMA 2006;296:2217-26.

20 Hernandez AF, Greiner MA, Fonarow GC, et al. Relationship between early physician follow-up and 30-day readmission among Medicare beneficiaries hospitalized for heart failure. JAMA 2010;303:1716-22.
21 Bhatia RS, Tu JV, Lee DS, et al. Outcome of heart failure with preserved ejection fraction in a population-based study. N Engl J Med 2006;355:260-9.

22 Owan TE, Hodge DO, Herges RM, et al. Trends in prevalence and outcome of heart failure with preserved ejection fraction. N Engl J Med 2006;355:251-9.

23 Lee DS, Austin PC, Stukel TA, et al. "Dose-dependent" impact of recurrent cardiac events on mortality in patients with heart failure. Am J Med 2009;122:162-9.

24 Setoguchi S, Stevenson LW, Schneeweiss S. Repeated hospitalizations predict mortality in the community population with heart failure. Am Heart J 2007;154:260-6.

25 Peacock WF, Braunwald E, Abraham W, et al. National Heart, Lung, and Blood Institute working group on emergency department management of acute heart failure: research challenges and opportunities. J Am Coll Cardiol 2010;56:343-51.

26 Krumholz HM, Amatruda J, Smith GL, et al. Randomized trial of an education and support intervention to prevent readmission of patients with heart failure. J Am Coll Cardiol 2002;39:83-9.

27 Phillips CO, Wright SM, Kern DE, et al. Comprehensive discharge planning with postdischarge support for older patients with congestive heart failure: a meta-analysis. JAMA 2004;291:1358-67.

28 Graff L, Orledge J, Radford MJ, et al. Correlation of the agency for health care policy and research congestive heart failure admission guideline with mortality: peer review organization voluntary hospital association initiative to decrease events (PROVIDE) for congestive heart failure. Ann Emerg Med 1999;34:429-37.

29 Smith WR, Poses RM, McClish DK, et al. Prognostic judgments and triage decisions for patients with acute congestive heart failure. Chest 2002;121:1610-17.

30 Lee DS, Stitt A, Austin PC, et al. Prediction of heart failure mortality in emergent care: a cohort study. Ann Intern Med 2012;156:767-75. 